\title{
Global Mental Health Resources and Services: A WHO Survey of 184 Countries.
}

\author{
Jodi Morris, $\mathrm{PhD},{ }^{1}$ \\ Antonio Lora, $\mathrm{MD},{ }^{2}$ \\ Ryan McBain, MSc, ${ }^{3}$ \\ Shekhar Saxena, $\mathrm{MD}^{1}$
}

\begin{abstract}
Data derived from the World Health Organization's (WHO) Mental Health Atlas Project 2011 are presented. These data provide the latest estimates on available resources for the treatment and prevention of neuropsychiatric disorders covering 98 percent of the world's population. Resources are defined in terms of governance, financing, mental health care delivery, human resources, essential medicines, and information systems. The Atlas project was initiated to guide policy and planning efforts in order to meet the large and growing burden of neuropsychiatric disorders worldwide. Results indicate that 60 percent of countries have a dedicated mental health policy; 71 percent possess a mental health plan; and 59 percent report having dedicated mental health legislation. Median mental health expenditures per capita are US\$1.63, with large variation among income groups, ranging from US\$ 0.20 in low income countries to US\$ 44.84 in high income countries. Globally, 67 percent of financial resources are directed towards mental hospitals. The global median number of facilities per 100,000 population were; 0.61 outpatient facilities, 0.05 day treatment facilities, 0.01 community residential facilities, and 0.04 mental hospitals. There are 7.04 psychiatric beds per 100,000 population in mental hospitals in comparison to 1.4 psychiatric beds per 100,000 population in general hospitals. Higher income countries typically report more facilities and higher admission/ utilization rates. Three quarters of patients admitted to mental hospitals remain there less than one year. There is a clear pattern whereby greater rates of human resources are observed in higher income countries. Globally, nurses represented the
\end{abstract}

\footnotetext{
${ }^{1}$ Department of Mental Health and Substance Abuse, World Health Organization, Geneva, Switzerland.

${ }^{2}$ Mental Health Department/Dipartimento di Salute Mentale, Lecco General Hospital/ Ospedale di Lecco, Lecco, Italy.

${ }^{3}$ Department of Global Health and Population, Harvard School of Public Health, Cambridge, USA.
}

Corresponding Author Contact Information: Jodi Morris at jodi.morris.phd@gmail.com; Department of Mental Health and Substance Abuse, World Health Organization, Geneva, Switzerland. 
most prevalent professional group working in the mental health sector. User and family associations are present in about two thirds of the countries, with greater representation in higher income countries. Results from Mental Health Atlas 2011 reinforce the urgent need to scale up resources within countries to meet the high and growing burden of mental disorders.

Keywords: Mental health systems, mental health services, human resources, policy, plans and legislation, information systems, WHO

Recommended Citation: Morris J, Lora A, McBain R, Saxena S. Global Mental Health Resources and Services: a WHO Survey of 184 Countries. Public Health Reviews. 2012;34: epub ahead of print.

\section{GLOBAL MENTAL HEALTH RESOURCES AND SERVICES: A WHO SURVEY OF 184 COUNTRIES.}

Neuropsychiatric disorders are estimated to contribute to 13 percent of the global burden of disease. ${ }^{1}$ Though the burden of mental disorders does not vary considerably across countries, recent research indicates that the availability of mental health resources is highly discrepant across countries. ${ }^{2}$ The resources needed vary from adequate infrastructure and suitably trained health personnel who can deliver good care, to legislative and policy initiatives that can provide the necessary governance and oversight to implement evidence-based care. . $^{3,4}$

Until recently, little information was available on worldwide mental health resources. In order to address this information gap, project Atlas was launched by the World Health Organization (WHO) in 2000 in an attempt to map mental health resources across the world. ${ }^{5}$ A subsequent version of Atlas has allowed for a comparison of the situation over time. ${ }^{6}$

The data presented in this paper are derived from WHO's 2011 edition of the Mental Health Atlas Project. ${ }^{7}$ A survey was sent to all WHO Member States and Associate Territories. Data were obtained from 184 of 193 Member States, covering 95 percent of WHO Member States and 98 percent of the world's population. In addition to global medians, results are presented by World Bank Income Group and WHO Regional Group, shortened in the text as AFR (Africa), AMR (Americas), EMR (Eastern Mediterranean), EUR (Europe), SEAR (South-East Asia) and WPR (Western Pacific).

Governance, financing, service delivery, human resources, medicines, and information systems are key building blocks of the mental health system in a country. ${ }^{8}$ Advancement in all these areas is necessary to improve mental health outcomes. Atlas 2011 permits a detailed analysis of these issues from a global perspective. 


\section{GOVERNANCE}

Governance is arguably the most complex and critical building block of any health system. It encompasses the role of the government in health care and its relation to other actors whose activities impact on health. Governance represents a continued process involving different components, from policy and legislation formation to financing and monitoring.

Mental health policies and plans are essential tools for outlining and enforcing the framework of the mental health system. A mental health policy may be broadly defined as an official statement of a government which conveys an organized set of values, principles, objectives and areas for action to improve the mental health of a population. Atlas 2011 indicates that a dedicated mental health policy is present in about six out of every ten countries-covering roughly 72 percent of the world's population. Among countries with such policies, three-quarters have been recently approved or updated since 2005. Aside from the majority of countries that have a dedicated mental health policy, a quarter possess general health policies which cover mental health issues, while one in ten countries do not have any policy that addresses aspects of mental health. Between 2005 and 2011, 21 countries acquired a new policy.

Mental health plans serve to delineate strategies and activities that will be implemented to meet policy objectives and typically specify elements such as budget and a time frame for implementation. They play a critical role in translating policy into practice. Mental health plans are present in almost three quarters of countries $(71 \%)$, and eight in every ten countries have formulated or updated their current plan since 2005. This evolution may be seen, in part, as a greater prioritization of mental health within the global health arena over the past ten years, as embodied in seminal publications such as the 2001 World Health Report. ${ }^{3}$ WHO strongly prompted countries to define and implement mental health policies and plans and this work was a major component of WHO technical collaboration in all the Regions.

Mental health legislation may cover a broad array of issues including access to mental health care and other services, quality of mental health care, admission to mental health facilities, consent to treatment, freedom from cruel, inhuman and degrading treatment, freedom from discrimination, the enjoyment of a full range of civil, cultural, economic, political and social rights, and provisions for legal mechanisms to promote and protect human rights (e.g., review bodies to oversee admission and treatment to mental health facilities, monitoring bodies to inspect human rights conditions in facilities and complaints mechanisms). Dedicated mental health legislation is present in about six countries out of every ten (59\%), and among countries 
with dedicated legislation, less than half $(42 \%)$ were enacted or revised since 2005. Legislation was initiated or revised in 2005 or later in 15 percent of the AFR countries, 11 percent of AMR countries, 40 percent of the EMR countries, 71 percent of EUR countries, 25 percent of SEAR countries, and 39 percent of WPR countries. Complete absence of legislation is rare: only one country in ten does not have either dedicated legislation or mental health legal provisions covered in other laws (e.g., laws on disability and welfare). From 2005 to 2011, a positive trend was observed: 35 countries implemented new mental health legislation (either dedicated or added to other laws).

On the whole, it is a positive result that the vast majority of policy and plan documents have been approved or updated since 2005, as was a similar majority of legislative documents since 2001. However, it should be noted that, although the presence of an updated policy or legislation is a necessary component for good governance, it is not sufficient to assure the quality of mental health care. In fact, Atlas 2011 data cannot capture whether legislation has been adequately implemented, or if a policy has effectively promoted practical changes in a country's mental health system.

\section{FINANCING}

Financing is a crucial building block of the mental health system. Without adequate financing, mental health policies and plans remain only in the realm of good intentions. ${ }^{3}$ Today, there are glaring inequalities between countries in terms of their public financing of mental health: median mental health expenditures per capita are US\$ 1.63 with large variation among income groups, ranging from US\$ 0.20 in low income countries to US\$ 44.84 in high income countries (Figure 1). The correlation between mental health spending per capita and gross national income per capita is robust $(\mathrm{r}=0.78)$; however, other factors such as level of human resources and presence of advocacy groups are also liable to influence the investment made in mental health.

In terms of overall mental health spending, the global median percentage of government health budget dedicated to mental health is 2.8 percent. Income also influences this proportion: lower income countries spend a smaller percentage of their health budget on mental health. The median percentage of health expenditures dedicated to mental health is 0.5 percent in low income countries and 5.1 percent in high income countries, with graduated values in lower-middle (1.9\%) and upper-middle income countries (2.4\%). This level of allocation is considerably higher in EUR and EMR and is lowest in AFR and SEAR. 


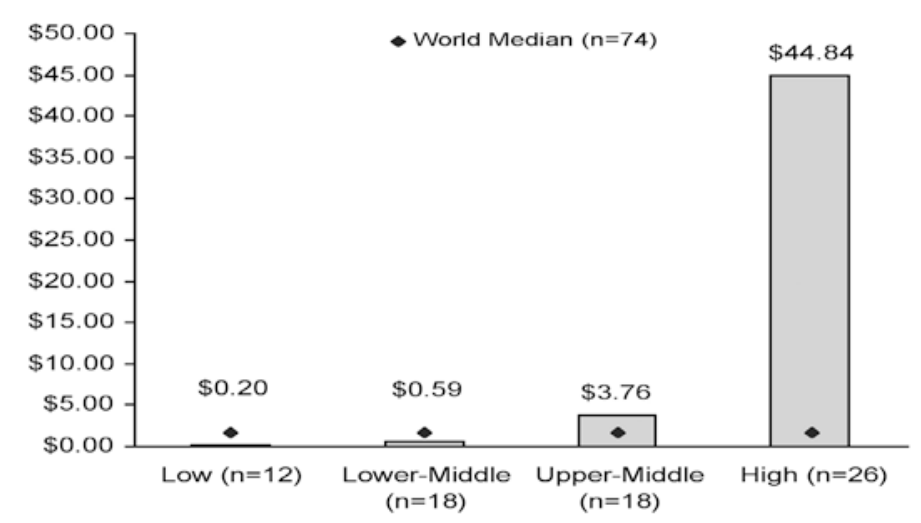

Fig. 1. Median mental health expenditures per capita by World Bank income group classification (see Table1). ${ }^{7}$

The WHO recommends the decentralization of mental health resources by shifting treatment from institutionalized care in mental hospitals to community-based care. ${ }^{3}$ Along these lines, the proportion of mental health expenditures allocated to mental hospitals serves as a proxy for the concentration and priority of institutionalized care in a country. Globally, 67 percent of financial resources are directed towards mental hospitals, indexing the relatively centralized nature of most countries' mental health systems. The percentage of mental health expenditures allocated to mental hospitals is consistent across low and middle income groups (73\%); however, it is slightly lower (54\%) in the high income group. The percentage of mental health expenditures on mental hospitals varies considerably across WHO regions with a low of 36 percent in EMR to a high of 77 percent in AFR.

\section{MENTAL HEALTH INFORMATION SYSTEMS}

Governance is not possible without monitoring and evaluation. The primary purpose of a mental health information system is not simply to gather data, but rather to enable decision-making that will lead to more effective governance and service improvement. ${ }^{9}$ A majority of countries collect data on the number of people treated and service user diagnoses at mental hospitals, general hospitals and outpatient facilities. In contrast, only a minority of countries collect these data from primary care facilities and community residential facilities. Data collection is only a first step; without the dissemination of data, there is a much lower likelihood that this 
information will be used to improve governance and service development. In terms of dissemination, approximately four countries out of every ten produce a report focused on mental health activities. However, such publications are less frequent in low and middle income countries. This result underscores the work that still remains to be done, as good governance requires the analysis and dissemination of information, as well as the inclusion of additional stakeholders to promote accountability.

\section{MENTAL HEALTH SERVICE DELIVERY}

\section{Primary care}

The integration of mental health into primary care has been a core recommendation of WHO for more than 30 years, but Atlas 2011 results indicate that this goal is far from being fully achieved. In terms of regulations and procedures, the majority of countries allow primary health care (PHC) doctors to prescribe and/or continue prescribing medicines for mental and behavioral disorders either without restrictions (56\%) or with some legal restrictions (40\%), such as allowing prescriptions only in certain categories of medicines or only in emergency settings. Three percent of respondent countries did not allow any form of prescription by PHC doctors. In contrast, 71 percent of countries do not allow nurses to prescribe or continue to prescribe these medicines; 26 percent allow nurses to prescribe with restrictions, and three percent to do so without restrictions. Prescription regulations for nurses are strongly influenced by income level: While only 27 percent of low income countries prohibit nurses from prescribing medicines, the majority of lower-middle income (70\%), upper-middle income (87\%) and high income (91\%) countries prohibit prescription by nurses. Furthermore, 13 percent of countries have an official policy or law enabling PHC nurses to independently diagnose and treat mental disorders within the primary care system. Such policies are also less common in high (7\%), upper-middle (8\%) and lower-middle (12\%) income countries in comparison with low income countries (29\%).

Regions in which a greater percentage of countries allow PHC doctors to prescribe psychotherapeutic medicines without restrictions include AMR (68\%) and AFR (61\%) in comparison to EMR (53\%), EUR (52\%), WPR $(48 \%)$ and SEAR (44\%). Regarding prescription by nurses, nine percent of AFR and four percent of WPR permit nurses to prescribe psychotherapeutic medicines without restrictions. No countries in AMR (0\%), EMR (0\%), 
EUR $(0 \%)$ and SEAR (0\%) allow such a practice. The lack of availability of psychiatrists as well as geographic barriers may play a role in whether countries permit PHC nurses to prescribe medicines for mental and behavioural disorders.

These regulations concerning primary care staff severely hamper accessibility of appropriate care for patients with mental disorders. In settings where there is a demonstrable scarcity of psychiatrists, it is necessary to allow primary care doctors to prescribe without restrictions and for nurses at least to continue prescriptions, if not also to prescribe with some restrictions. In order to bridge the gap between prevalence of mental disorders in the community and access to appropriate care, restrictive regulations in the primary care setting should be modified. The WHO mental health Gap Action Programme (mhGAP) $)^{10}$ underscores the role of primary care staff, doctors and nurses in treating not only mild and moderate mental health disorders, but also more severe manifestations of mental illness.

Primary care and mental health services more broadly are linked by the establishment of formalized referral procedures. Atlas 2011 documents the existence of official referral procedures with mental health services in three quarters $(76 \%)$ of the countries and back-referral in two thirds $(65 \%)$. Though these proportions are substantial, the extent to which these procedures are followed is unknown.

Training on mental health issues is weak in primary care settings: in only a quarter (28\%) of countries have the majority of PHC doctors (greater than $50 \%$ ) received official in-service training on mental health issues within the last five years; this figure is lower (22\%) for PHC nurses. Percentages did not vary considerably by income level, indicating that the existing knowledge gap is not primarily reflective of a country's inability to pay for training. A related issue pertains to knowledge transfer to primary care staff. Manuals outlining diagnosis and treatment protocols provide an entry point for training. It is therefore disconcerting that only one third of countries (36\%) have officially approved manuals on the management and treatment of mental disorders at a majority of PHC clinics.

Furthermore, merely having information available at facilities is not enough to ensure that individuals learn and apply institutional knowledge. A more in-depth analysis is required to understand how and why specific diagnostic procedures and manuals are effective, how many patients are referred from one sector to another and which health outcomes they produce. Along these lines, a recent analysis looking at the integration of mental health treatment into primary care found poor integration among sectors and discussed lack of integration as a major obstacle to scaling up services in low and middle income countries. ${ }^{11}$ 


\section{Specialized mental health services}

Mental health services are a foundational component of all mental health systems. An outpatient facility is a facility that specifically focuses on the management of mental disorders and the clinical problems related to it on an outpatient basis in a community setting. Outpatient facilities exist in most countries, with only nine countries worldwide reporting an absence of these facilities. Globally, there are 0.61 outpatient facilities per 100,000 population. The availability of facilities by income group follows a clear pattern, with the median rate of facilities in high income countries 58 times greater than in low income countries. Furthermore, many low income and lower-middle income countries have only the most rudimentary network of these facilities.

The median annual rate of service users treated in outpatient clinics per 100,000 population is 384 , with substantial variability by country income level (Figure 2). Accessibility, in terms of the rate of treated outpatients, is six times greater in high income countries as compared to lower-middle income countries, and thirty-eight times higher than in low income countries. Similarly, the rate of outpatient contacts in high income countries is ten times higher than in lower-middle income countries and fifty times higher than in low income countries. A substantial variability exists by region, ranging from 80 outpatients per 100,000 population in AFR to 1,926 outpatients per 100,000 population in EUR.

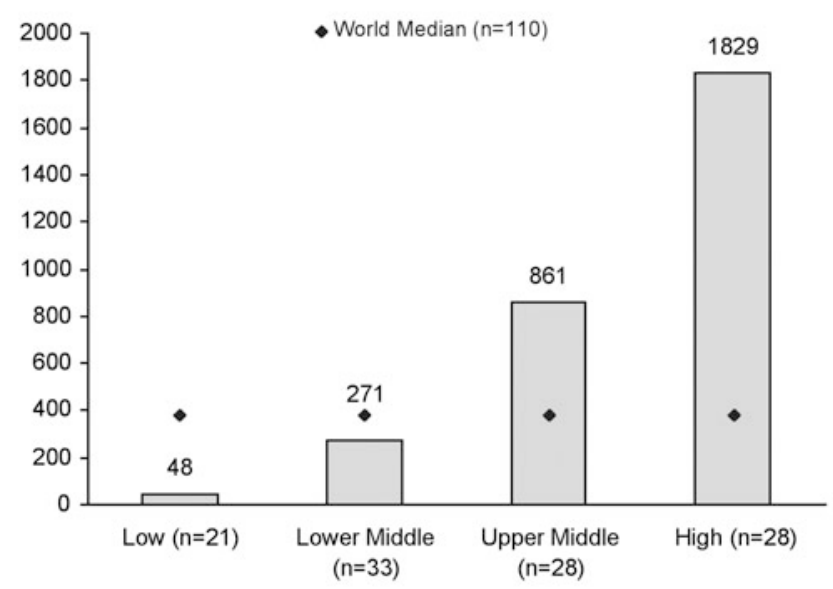

Fig. 2. Rate of persons seen in mental health outpatient facilities per 100,000 population by World Bank income group classification. ${ }^{7}$ 
Looking at the process of care, only 32 percent of countries have a majority of facilities that provide follow-up care. This figure varies across income classifications; seven percent of low income, 29 percent of lowermiddle income, 39 percent of upper-middle income, and 45 percent of high income countries provide follow-up care at a majority of facilities. By region, EUR has the greatest percentage of countries in which a majority of facilities provide follow-up community care $(50 \%)$, and EMR has the smallest percentage $(6 \%)$. However, it should be noted that the definition of follow-up community care may differ by country.

Psychosocial interventions use primarily psychological or social methods for the treatment and/or rehabilitation of a person with a mental disorder or for the substantial reduction of psychosocial distress. Only 44 percent of countries have a majority of facilities which provide psychosocial interventions, a figure which also varies by income classification; 14 percent of low income, 34 percent of lower-middle income, 61 percent of upper-middle income, and 59 percent of high income countries provide psychosocial care at a majority of facilities. In AMR and EUR, 64 percent and 59 percent of countries have a majority of facilities providing psychosocial interventions, respectively. In contrast, 24 percent of countries in AFR and 25 perecnt of countries in EMR and SEAR have a majority of facilities providing such care.

Outpatient facilities play a crucial role in service delivery, providing a primary point of care in community settings. As a gatekeeper to communitybased access to mental health care, the continued expansion of outpatient facilities is vital to the improvement of accessibility and to reduce the treatment gaps for mental disorders. Follow-up home visits and psychosocial interventions are compulsory ingredients in community care, but follow-up care is widespread in only one third of countries, and psychosocial interventions are regularly provided in only four countries out of every ten. These shortages are concentrated in both low and lower-middle income countries. Without these active ingredients, community care is impoverished, the responsiveness of the mental health system is weakened and the transfer of patients with severe mental illness from mental hospitals to community settings is hindered.

A day treatment facility provides care for service users during the day. The median rate of day treatment facilities per 100,000 population is 0.05 . In 26 percent of countries, day treatment facilities do not exist-particularly in low and lower-middle income countries. When analysed by income level, variation in treatment rates at day treatment facilities is considerable: the median treatment rate is zero persons per 100,000 people in low income 
countries, one person in lower-middle income countries, four persons in upper-middle income countries and 44 persons in high income countries. Regional variation in day treatment facilities is even more pronounced: where 43 persons per 100,000 population are treated in day treatment facilities in EUR countries, the next highest rate of treatment, represented by AMR, is approximately 50 times smaller.

A community residential facility is a non-hospital, community-based mental health facility that provides overnight residence for people with mental disorders. Community residential facilities are present only in one half $(54 \%)$ of countries-mainly high income countries. This fact is reflected in the median rate of beds and patients treated at such facilities: These rates are markedly greater in high income countries (respectively 10.15 per 100,000 and 5.8 per 100,000) as compared with low, lowermiddle and upper-middle income countries, all of which have a median rate of zero beds and residents per 100,000 population. The number of residential facility beds per 100,000 population varies more substantially from region to region, with EUR and SEAR having the highest median rates, at 2.60 and 0.78 per 100,000 population, respectively, and AFR and WPR having the lowest, both at 0.00 per 100,000 population.

It is unclear whether the scarcity of community residential facilities in low and middle income countries is due to a shortage of resources, or if these facilities are inappropriate for low and middle income settings. Day treatment facilities play an important role in Western countries and in larger cities in low and middle income countries, but they may not be as useful because of distances or culturally accepted in rural low and middle income regions. ${ }^{12}$ Rehabilitation in the community through home visits may be a valid alternative. In order to resolve these questions, health system research should provide further clarity in coming years.

Overall, mental hospitals still represent the primary mode of inpatient service: they are present in 80 percent of countries. A mental hospital is a specialized hospital-based facility that provides inpatient care and long-stay residential services for people with mental disorders. Usually these facilities are independent and standalone, although they may have some links with the rest of the health care system. Countries where mental hospitals do not exist include small country-islands in the Americas and the Western Pacific region, ten African countries, and some European countries, such as Iceland, Italy and Sweden. The absence of mental hospitals is due to different causes. In European high income countries, the process of deinstitutionalization has resulted in community-based systems of care operating exclusively, while in countries with very small populations the utility of such facilities 
is limited. Globally, there are 7.04 beds per 100,000 population, while the rate of admissions to mental hospitals is 39.3 per 100,000. Rates of mental hospital beds and admissions both show a trend related to income (Figure 3 and Figure 4), with the rate of beds being 30 times greater in high income countries as compared to low income countries, and 20 times greater admission levels. There is also a significant regional variability in the rate of beds in mental hospitals; globally, there are 7.04 beds per 100,000 population, but this figure ranges from 0.9 in SEAR to 39.4 in EUR.

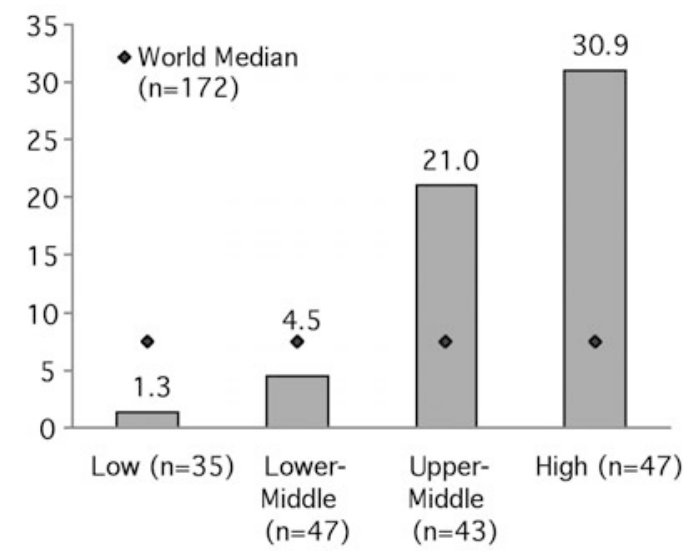

Fig. 3. Median rate of mental hospital beds per 100,000 population by World Bank income group classification. ${ }^{7}$

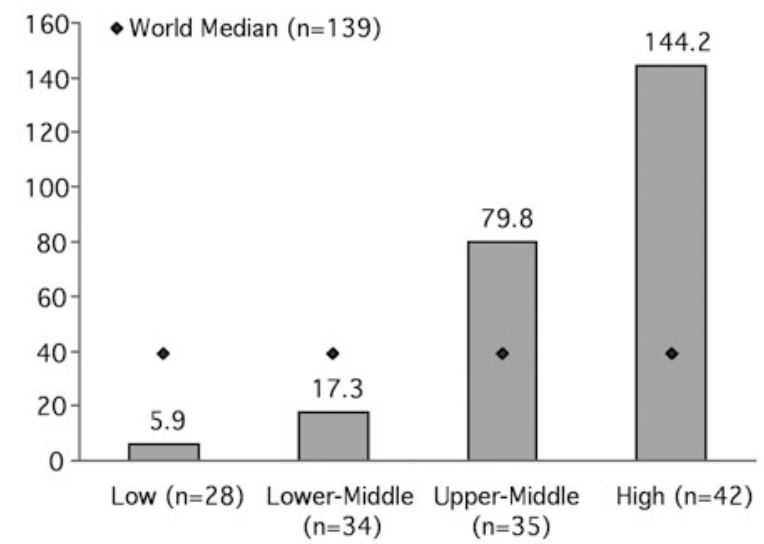

Fig. 4. Median admissions to mental hospitals per 100,000 population by World Bank income group classification. ${ }^{7}$ 
The process of deinstitutionalization can be observed by comparing data from 2005 and 2011. Globally, the median decrease in mental hospital beds was -0.11 per 100,000 population, indicating that the majority of countries reduced their rate of mental hospital beds over this period. This would imply that, in a country with a population of ten million people, there would be an expected decrease of 11 beds over this period. Beds in mental hospitals decreased mainly in high income (-0.43) and upper-middle income countries (-0.90), as compared with lower-middle (-0.14) and low income countries (-0.01). In looking at the change in mental hospital beds by region, AMR and EUR showed the greatest decreases. In WPR, the median rate of change was zero, indicating that in the majority of the countries there was either no change or an increase in the rate of beds. In all other regions, more countries decreased than increased.

A significant majority (77\%) of individuals admitted to mental hospitals remain less than one year. However, this also implies that almost a quarter (23\%) of people admitted to mental hospitals remain longer than a year after admission. This value varies by income group: the median percentage of individuals admitted to mental hospitals who remain for less than one year is 95 percent in low income countries; 77 percent in lower-middle income countries; 67 percent in upper-middle income countries; and 71 percent in high income countries. The use of mental hospitals seems to differ by income: in low income countries, mental hospitals may function more often as acute mental health wards, whereas in more affluent countries their role is closer to residential units for long-stay patients. This difference provides information for planning the downsizing of mental hospitals. In general, in low income countries, the planners may need to focus on developing community-based inpatient units, while in middle and high income countries the development of community residential facilities is needed.

Psychiatric wards in general hospitals are present in 85 percent of countries. While the global median rate of beds in psychiatric wards is 1.4 per 100,000 population, low and lower-middle income countries have similar median rates of psychiatric beds in general hospitals (around 0.5 beds per 100,000), and higher rates are observed in upper-middle ( 2.7 beds per 100,000 population) and high income $(13.6$ beds per 100,000$)$ countries. All WHO regions other than EUR (10.5 beds per 100,000) have less than two beds per 100,000 people.

Globally, the median rate of the admissions in general hospitals is 24.2 per 100,000 population. By income group, low and lower-middle income countries have similarly low annual rates (around 6 admissions per 100,000 
population), with upper-middle income countries being substantially higher (36.6 per 100,000 population). High income countries have median rates $(175.4$ per 100,000$)$ that are almost 30 times greater than the low and lowermiddle income countries. Across regions, only WPR and EUR were higher than the global median, with the rate in EUR $(135$ per 100,000) being more than five times this figure. From 2005 to 2011 we did not observe any increase in the rate of general hospital beds.

Worldwide, two thirds (62\%) of mental health beds are placed in mental hospitals, one fifth (21\%) in general hospital units and one sixth (16\%) in community residential facilities. Thus, the main resource for admission of persons with acute disorders cases is still mental hospitals.

There is a global trend towards reduction in psychiatric beds. On the one hand, an observed decrease of resources spent on mental hospitals represents a positive change, whereby resources are being decentralized. On the other hand, a paucity of general hospital psychiatric beds do not suffice to replace mental hospitals in caring for acute mental disorders. In the coming years, it will be important to monitor this balance as the role of community-based facilities increases.

\section{HUMAN RESOURCES}

Mental health personnel are the most valuable resource within the mental health system, ${ }^{13,14}$ and the current paucity of mental health professionals within low and middle income countries is a major obstacle to providing care for people with mental disorders. Although PHC staff can and should also provide mental health care, adequate numbers of specialized mental health professionals are still essential. Atlas 2011 results confirm that the majority of countries lack adequate numbers of mental health professionals, most notably in low income countries. Across all professions, the global median rate of human resources working in the mental health sector is 10.7 workers per 100,000 population. By region, EUR consistently has the highest rates of human resources (43.9), and AFR has the lowest (1.7).

Globally, nurses (psychiatric and non-psychiatric) represent the largest professional group working in the mental health sector. The median rate of nurses in this sector, 5.8 per 100,000, is greater than the median rate of all other human resources groups combined. There is a clear trend in the rate of human resources by income group, with greater rates associated with higher income group classifications (Figure 5). 


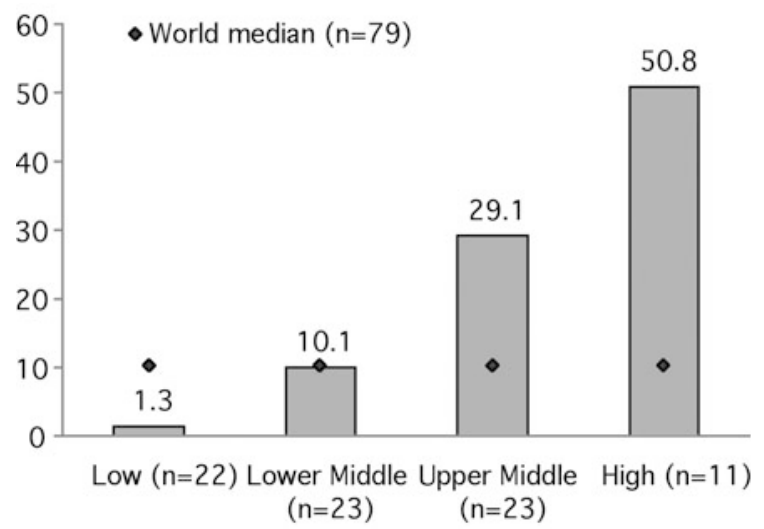

Fig. 5. Median rate of human resources for mental health per 100,000 population by World Bank income group classification. ${ }^{7}$

While the rate of social workers and occupational therapists differs only between high income countries and other income group classifications, disparities in the number of doctors, nurses and psychologists are much more pronounced across all four income group classifications (Table 1). The largest difference is in the rate of psychologists and psychiatrists, which are over 100 and 180 times greater (respectively) in high income compared with low income countries.

\section{Table 1}

Current level of health professionals working in the mental health sector by World Bank income group classification ${ }^{7}$

\begin{tabular}{c|c|c|c|c|c|c}
\hline $\begin{array}{c}\text { Income } \\
\text { Group }\end{array}$ & Psychiatrists & $\begin{array}{c}\text { Other } \\
\text { Medical } \\
\text { Doctors }\end{array}$ & Nurses & Psychologists & $\begin{array}{c}\text { Social } \\
\text { Workers }\end{array}$ & $\begin{array}{c}\text { Occupational } \\
\text { Therapists }\end{array}$ \\
\hline $\begin{array}{c}\text { Low } \\
(\mathrm{n}=25-38)\end{array}$ & 0.05 & 0.06 & 0.42 & 0.02 & 0.01 & 0.00 \\
\hline $\begin{array}{c}\text { Lower- } \\
\text { middle } \\
(\mathrm{n}=31-52)\end{array}$ & 0.54 & 0.21 & 2.93 & 0.14 & 0.13 & 0.01 \\
\hline $\begin{array}{c}\text { Upper- } \\
\text { middle } \\
(\mathrm{n}=26-42)\end{array}$ & 2.03 & 0.87 & 9.72 & 1.47 & 0.76 & 0.23 \\
\hline $\begin{array}{c}\text { High } \\
(\mathrm{n}=26-47)\end{array}$ & 8.41 & 1.49 & 29.15 & 3.79 & 2.16 & 1.51 \\
\hline
\end{tabular}


Such analyses indicate the need for developing the capacity of other mental health professionals to deliver effective community interventions. For example, the role of nurses in mental health is continuing to evolve. ${ }^{15}$ In many countries, nurses have become expert case managers in severe mental illnesses and many evidence based interventions could be delivered directly by them. Mental health care providers and policy makers should consider how task shifting and incentives might improve productivity. Task shifting involves delegating tasks to existing or new professionals with either less training or narrowly focused training to increase access to lower costservices. For example, in mental health settings, task shifting might include transferring tasks from a psychiatrist to a nurse or a non-specialist doctor. ${ }^{13,14}$

In two thirds $(64 \%)$ of countries, all or almost all psychiatrists work in public settings, and in only one country in ten do psychiatrists work predominately in private practice. This may be considered a positive element insofar as the absence of a public network could inhibit response capacity to severe mental illnesses. However, one must also note that in 30 percent of countries all psychiatrists work in mental hospitals, as do 38 percent of nurses in the mental health sector. This absence of mental health staff outside mental hospitals severely damages mental health care in terms of accessibility, appropriateness, human rights and efficiency.

Attention should be paid not only to current mental health staff, but also to future mental health professionals - those that are graduating from training. At the global level, there are more graduates with degrees in nursing (5.15 per 100,000 population) than in any other health profession working in the field of mental health. After nurses, the most common health professional graduates are medical doctors (3.38 per 100,000 population). Comparatively, there is a much smaller pool of psychologists, psychiatrists, social workers and occupational therapists who graduated in the past academic year. The shortage in the number of mental health professionals graduating varies by country income level and region (in Africa, the number of graduates is one fourth the global median). Strategic planning in this area should involve not only the mental health sector, but also other health sectors and governmental bodies.

Family and user associations represent human resources that are important allies in fighting for the health care and rights of people with mental disorders. User and family associations are present in 64 percent and 62 percent of countries, respectively. User associations are more prevalent in higher income countries - in 83 percent of high income countries versus 49 percent of low income countries - as are family associations, which are present in 80 percent of high income countries and 39 percent of low income countries. 
However, even in upper-middle income countries, these associations have only a few members, and their interactions with governmental bodies are limited. In only 40 percent of countries are users/families associations routinely involved in formulation or implementation of mental health policies at the national level.

\section{CONCLUSIONS}

The role of information is usually underestimated in mental health systems, not only in low and middle income countries but also in high income countries. High quality information is needed to monitor and to change the mental health system. The WHO confirms its commitment to use information for reducing the mental health treatment gap, for developing community care, and for strengthening mental health systems.

The WHO Atlas 2011 provides basic information to advocate for scaling up mental health care in low and middle income countries. It does this in several ways. First, Atlas 2011 highlights the urgent need for more resources in order to provide for currently unmet needs in mental health. Second, it suggests that limited resources should be better used, i.e., redirecting resources from institutional to community-based care. Third, Atlas supports community care through the provision of information. The reform of mental health systems is not merely a matter of resources, but also implicates cultural and scientific perceptions and practices among mental health professionals, health managers and politicians. Evidence indicates that, on the one hand, 80 percent of resources for mental health are spent in mental hospitals; on the other hand, reduction in the treatment gap necessitates an expansion of community-based care. Statistics such as these should not only stimulate scientific debate on mental health systems, but compel greater prioritization of the global mental health agenda.

One of the shortcomings of the Atlas project is that it only investigates the differences between countries and not intra-country disparities. These disparities are not only related to social differences, but also to geographic ones (e.g., urban versus rural). Atlas is not the right tool to monitor these differences; WHO Assessment Instrument for Mental Health Systems (WHO-AIMS), ${ }^{16,17}$ a WHO indicator system for evaluating mental health systems in low and middle income countries, is a more appropriate tool to provide such information.

Good information does not necessarily drive decisions, but it guides them. Recent WHO initiatives help to bridge the gap between this information and public health action. The Mental Health Gap Action 
Programme (mhGAP) is WHO's action plan to scale up services for mental, neurological and substance use disorders for countries especially with low and lower-middle incomes. The goal of this programme is not only to reinforce the commitment of governments, international organizations, and other stakeholders to increase the allocation of financial and human resources for care of mental disorders, but also to achieve much higher coverage with key interventions in the countries with low and lower-middle incomes that have a large proportion of the global burden of mental disorders. Moreover, at the recent 130th Executive Board meeting of the WHO, a new resolution "Global Burden of Mental Disorders and the Need for a Comprehensive, Co-ordinated Response from Health and Social Sectors at the Country Level (EB 130.R8)" was adopted by Member States. It strengthens the response to mental health needs at a global level, urging Member States to prioritize mental health in the public health agenda and requesting WHO to provide a comprehensive mental health action plan.

Mental health needs a public health approach given the interdependence of mental and physical health and the growing burden of mental disorders worldwide, especially in poor countries. The WHO agenda for mental health strongly promotes a public health approach: it addresses the mental health of the population, it focuses on assisting low and middle income countries to develop mental health systems and services that focus on community care, advocate for prevention and promotion, and integrate primary and specialized care. Greater integration of mental health into the wider public health agenda is needed and more public health "thinking" should be used in mental health planning, looking not only at care but also to prevention and promotion. ${ }^{18}$

\section{Acronyms List:}

$\mathrm{AFR}=$ Africa Region

$\mathrm{AMR}=$ Americas Region

$\mathrm{EMR}=$ Europe Region

PHC $=$ Primary health care

SEAR $=$ South-East Asia Region

WPR $=$ Western Pacific Region

Conflicts of Interest: None declared.

About the Authors: Jodi Morris holds a PhD in Psychology from Boston College and completed her clinical training at Harvard Medical School (Cambridge Hospital). She served for seven years as a technical officer in the Department of Mental Health and Substance Abuse at WHO in Geneva, Switzerland where she acted as overall Project Manager for the Atlas study. She recently has taken up a faculty position at the University of British Columbia (Okanagan campus) in Canada. 
Antonio Lora is Director of the Mental Health Department of Lecco General Hospital in Lecco, Italy and the Mental Health Services Evaluation Advisor to the Regione Lombardia. He received his MD from Milano University and is the past President of the Italian Society of Psychiatric Epidemiology (SIEP). He served as a Senior Consultant to the Atlas study.

Ryan McBain holds a MSc from Harvard University and is a DS candidate in the Department of Global Health and Population at the Harvard School of Public Health. He is employed as a researcher at the François-Xavier Bagnoud Center for Health and Human Rights, where he studies the psychological effects of war trauma on children and adolescents and is leading an analysis on the cost-effectiveness of a behavioral intervention for these individuals. He served as a Consultant to the Atlas study.

Shekhar Saxena is Director of the Department of Mental Health and Substance Abuse at World Health Organization, Geneva. He is a psychiatrist by training with about 30 years of experience in research and programme management service, delivery and information systems in the areas of mental health, especially in low and middle income countries. He is responsible for implementation of WHO's mental health Gap Action Programme and he provided the overview vision and guidance for the Atlas project at each step.

\section{REFERENCES}

1. World Health Organization. The global burden of disease--2004 update. WHO: Geneva; 2008.

2. Saxena S, Thornicroft G, Knapp M, Whiteford H. Resources for mental health: scarcity, inequity, and inefficiency. Lancet. 2007;370:878-89.

3. World Health Organization. The world health report 2001 - mental health: new understanding, new hope. WHO: Geneva; 2001.

4. Saraceno B. Mental health: scarce resources need new paradigms. World Psychiatry. 2004;3:3-5.

5. World Health Organization. Atlas: mental health resources in the world 2001. WHO: Geneva; 2001.

6. World Health Organization. Atlas: mental health atlas 2005. WHO: Geneva; 2005.

7. World Health Organization. Atlas: mental health atlas 2011. WHO: Geneva; 2011.

8. World Health Organization. Everybody's business: strengthening health systems to improve health outcomes. WHO: Geneva; 2007.

9. World Health Organization. Mental health policy and service guidance package: monitoring and evaluation of mental health policies and plans. WHO: Geneva; 2005. 
10. World Health Organization. WHO Mental Health Gap Action Programme (mhGAP). WHO: Geneva. Available from URL: http://www.who.int/mental_ health/mhgap/en/index.html (Accessed 8 September, 2011).

11. World Health Organization/Wonca Joint Report. Integrating mental health in primary care - a global perspective. WHO: Geneva; 2007.

12. Saraceno B, van Ommeren M, Batniji R, Cohen A, Gureje O, Mahoney J, et al. Barriers to improvement of mental health services in low-income and middleincome countries. Lancet. 2007;370:1164-74.

13. Scheffler RM, Saxena S, Bruckner T, Yoon J, Shen G, Chisolm D, et al. Human resources for mental health: workforce shortages in lower and middle income countries. WHO: Geneva; 2011.

14. Bruckner TA, Scheffler RM, Shen G, Yoon J, Chisholm D, Morris J, et al. The mental health workforce gap in low- and middle-income countries: a needsbased approach. Bull World Health Organ. 2011;89:184-94

15. World Health Organization. Atlas: nurses in mental health 2007. WHO: Geneva; 2007.

16. World Health Organization. World Health Organization Assessment Instrument for Mental Health Systems (WHO-AIMS). WHO: Geneva; 2005. Available from URL: http://www.who.int/mental_health/evidence/WHO-AIMS/en/index. html (Accessed 26 June, 2012).

17. Saxena S, Lora A, van Ommeren M, Barrett T, Morris J, Saraceno B. WHO's Assessment Instrument for Mental Health Systems: collecting essential information for policy and service delivery, Psychiatr Serv. 2007;58:816-21.

18. Saraceno B, Freeman M, Funk M. Public mental health. In: Oxford Textbook of Public Health - 5th edition. Oxford University Press: Oxford; 2009. 